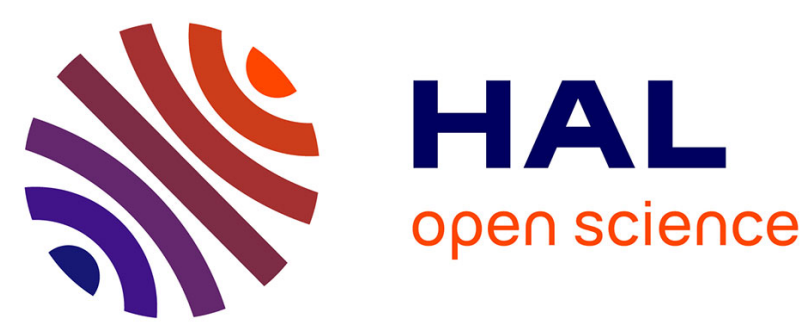

\title{
Correlative transmission electron microscopy and high-resolution hard X-ray fluorescence microscopy of cell sections to measure trace element concentrations at the organelle level
}

\author{
Vanessa Tardillo- Suarez, Benoit Gallet, Mireille Chevallet, Pierre-Henri \\ Jouneau, Rémi Tucoulou, Giulia Veronesi, Aurélien Deniaud
}

\section{To cite this version:}

Vanessa Tardillo- Suarez, Benoit Gallet, Mireille Chevallet, Pierre-Henri Jouneau, Rémi Tucoulou, et al.. Correlative transmission electron microscopy and high-resolution hard X-ray fluorescence microscopy of cell sections to measure trace element concentrations at the organelle level. Journal of Structural Biology, 2021, 213 (3), pp.107766. 10.1016/j.jsb.2021.107766 . hal-03338415

\author{
HAL Id: hal-03338415 \\ https://hal.science/hal-03338415
}

Submitted on 15 Sep 2021

HAL is a multi-disciplinary open access archive for the deposit and dissemination of scientific research documents, whether they are published or not. The documents may come from teaching and research institutions in France or abroad, or from public or private research centers.
L'archive ouverte pluridisciplinaire HAL, est destinée au dépôt et à la diffusion de documents scientifiques de niveau recherche, publiés ou non, émanant des établissements d'enseignement et de recherche français ou étrangers, des laboratoires publics ou privés. 


\section{Correlative transmission electron microscopy and high-resolution}

hard X-ray fluorescence microscopy of cell sections to measure trace element concentrations at the organelle level

Vanessa Tardillo Suárez ${ }^{\mathrm{a}, \#}$, Benoit Gallet ${ }^{\mathrm{b}, \#}$, Mireille Chevallet ${ }^{\mathrm{c}}$, Pierre-Henri Jouneau ${ }^{\mathrm{d}}$, Rémi Tucoulou $^{\mathrm{a}}$, Giulia Veronesi ${ }^{\mathrm{a}, \mathrm{c} *}$ and Aurélien Deniaud ${ }^{\text {** }}$

\section{Affiliations}

a ESRF, The European Synchrotron. 71 avenue des Martyrs, 38000 Grenoble, France

b Institut de Biologie Structurale, CEA, CNRS, Univ. Grenoble Alpes, 71 avenue des Martyrs, F-38042

Grenoble, France

c Univ. Grenoble Alpes, CNRS, CEA, IRIG, Laboratoire de Chimie et Biologie des Métaux, F-38000

Grenoble, France

d Univ. Grenoble Alpes, CEA, IRIG, MEM, F-38000 Grenoble, France

\# These authors contributed equally to this work

* Correspondence should be addressed to Dr Giulia Veronesi

giulia.veronesi@cea.fr

or to Dr Aurélien Deniaud

aurelien.deniaud@cea.fr 


\section{Abstract}

Metals are essential for life and their concentration and distribution in organisms are tightly regulated. Indeed, in their free form, most transition metal ions are toxic. Therefore, an excess of physiologic metal ions or the uptake of non-physiologic metal ions can be highly detrimental to the organism. It is thus fundamental to understand metal distribution under physiological, pathological or environmental conditions, for instance in metal-related pathologies or upon environmental exposure to metals. Elemental imaging techniques can serve this purpose, by allowing the visualization and the quantification of metal species in tissues down to the level of cell organelles. Synchrotron radiationbased X-ray fluorescence (SR-XRF) microscopy is one of the most sensitive techniques to date, and great progress was made to reach nanoscale spatial resolution. Here we propose a correlative method to couple SR-XRF to electron microscopy (EM), with the possibility to quantify selected elemental contents in a specific organelle of interest with $50 \times 50 \mathrm{~nm}^{2}$ raster scan resolution. We performed EM and SR-XRF on the same section of hepatocytes exposed to silver nanoparticles, in order to identify mitochondria through EM and visualize Ag co-localized with these organelles through SR-XRF. We demonstrate the accumulation of silver in mitochondria, which can reach a 10 -fold higher silver concentration compared to the surrounding cytosol. The sample preparation and experimental setup can be adapted to other scientific questions, making the correlative use of SR-XRF and EM suitable to address a large panel of biological questions related to metal homeostasis.

Keywords: X-ray fluorescence microscopy; Transmission electron microscopy; Hepatocytes; Mitochondria; Silver. 


\section{Introduction}

Metal ions are essential for Life. They are required for various processes such as redox-dependent enzymatic reactions, respiration or protein domain structuring. In addition, redox-active free metal ions can induce oxidative stress in cells. Therefore, living organisms tightly control metal ion uptake, intracellular trafficking, storage, and excretion. Although these complex mechanisms, termed homeostasis, are generally well understood for $\mathrm{Zn}, \mathrm{Fe}$ and $\mathrm{Cu}$ in humans, some molecular details remain unclear, in particular the biogenesis of FeS clusters in mitochondria (Beilschmidt et al., 2017). Moreover, disease-related metal overload or environmental exposure to metals or metal nanoparticles disrupt these mechanisms, altering cellular physiology. It is thus of paramount importance to be able to visualize trace element subcellular distribution, i.e. down to the organelle level for eukaryote cells.

Various approaches are used to study metal distribution within biological samples, with a large range of sensitivities and spatial resolutions (for review (Decelle et al., 2020)). For large field of view analyses with a moderate sensitivity, two methods exist: Laser-Induced Breakdown Spectroscopy (LIBS) and Laser Ablation Inductively Coupled Plasma Mass Spectrometry LA-ICP-MS (Busser et al., 2018; Greenhalgh et al., 2020; Wiemann et al., 2017). Their micrometer resolution makes them useful for the analysis of tissues. Tens of nanometer resolution can be reached with secondary ion mass spectroscopy (SIMS) and Energy-dispersive X-ray spectroscopy (EDX) in an electron microscope (for a general review on the different methodologies, see (Dressler et al., 2018)). However, their sensitivities do not allow the detection of molecular species at biologically relevant concentrations. The method of choice to analyze the distribution of any element of interest with subcellular resolution, sensitive enough to detect trace elements even in their soluble forms, is X-ray fluorescence (XRF) imaging performed in a synchrotron X-ray nanoprobe (Deng et al., 2017; Fus et al., 2019; Hasna et al., 2019; Tardillo Suárez et al., 2020). As of today, high-resolution 2D elemental images have most often been obtained on whole cells, where the XRF signal from the whole thickness of the cell is summed up, losing the in-depth resolution and consequently the precise distribution within the cell down to the organelle level. In order to overcome this limitation, 3D tomographic XRF imaging should be used (de Jonge et al., 2010; Fus et al., 2019; Yuan et al., 2013). However, the long acquisition time needed to collect a number of projections that allow the accurate reconstruction of the cell volume prevents a routine use of this technique in biology. Possible solutions applied so far consist in either performing 2D XRF imaging over a large sample set, then confirming the findings on a few specimens by acquiring 3D data (Fus et al. 2019), or in coupling 
2D XRF on whole cells with other techniques that allow the localization of subcellular compartments. Among the latter, it is worth mentioning organelle labelling followed by fluorescence microscopy (Carmona et al., 2019; Yuan et al., 2013), or TEM observations of cell sections (Chen et al., 2006; Veronesi et al., 2016). In this context, the possibility to perform correlative imaging of cell sections by $\mathrm{EM}$ and XRF is highly appealing. This requires the development of sample preparation and measurement strategies that enable the observation of the same specimen with both techniques, in spite of their different experimental requirements. For instance, EM observations rely on heavy metal staining of the biological samples, whereas staining is not recommended for XRF imaging, in particular with metals that would interfere with the XRF signal from the native elements.

Recently, we were able to observe cell sections by both TEM and XRF imaging, in order to follow the entry of $\mathrm{Ag}(\mathrm{I})$ ions released from AgNPs into the nuclei of hepatocytes (Tardillo Suárez et al., 2020). In the following, we present a correlative setup to push the combination of these techniques to its utmost performance by performing them on the same cell section, demonstrate that it is able to localize smaller organelles such as mitochondria ( $1 \mu \mathrm{m}$ large), and discuss the details of our sample preparation protocol. The latter is strongly dependent on the scientific question under study: in the case of second row transition metals (as $\mathrm{Pd}, \mathrm{Ag}, \mathrm{Cd}$ ) in biological samples, the X-ray excitation energy needed to achieve the highest detection capability for exogenous metal is in the order of $25 \mathrm{keV}$, much higher than the X-ray energy that would maximize the detection of native trace metals $(\mathrm{Mn}, \mathrm{Fe}, \mathrm{Cu}, \mathrm{Zn})$ and lighter physiological elements $(\mathrm{P}, \mathrm{S}, \mathrm{Cl}, \mathrm{K}, \mathrm{Ca})$. Therefore, if the energy is chosen in such a way that the detection of exogenous metal is optimized and its diluted ionic species can be detected, the SR-XRF signal from the native elements would remain weak. Considering this, we used $\mathrm{OsO}_{4}$ staining to obtain contrast of the cellular membranes in both SR-XRF and EM to visualize cell anatomy. A recent publication described a sample preparation strategy that used tannic acid in order to enhance the contrast in EM while avoiding heavy-metal staining, which proved its effectiveness for the detection of native metals at an X-ray energy of $10 \mathrm{keV}$ (Kashiv et al., 2016).

In this study, the choice of the experimental conditions and of the sample preparation strategy is driven by the need to detect ionic silver species, and is optimized for the study of the fate of second row transition metals in cells or tissues, as for instance in metallodrugs, drug delivery, and nanoparticle research. Ag is a non-physiological metal, but the increasing use of AgNPs as biocides in consumer products and medical devices causes humans to be exposed and to accumulate Ag species in their 
bodies. The binding properties of $\mathrm{Ag}(\mathrm{I})$ are similar to those of $\mathrm{Cu}(\mathrm{I})$, thus $\mathrm{Ag}(\mathrm{I})$ can take the place of $\mathrm{Cu}(\mathrm{I})$ in Cu-dependent enzymes, thus leading to their inactivation (Ciriolo et al., 1994). We also showed that $\mathrm{Ag}(\mathrm{I})$ can replace $\mathrm{Zn}(\mathrm{II})$ in the $\mathrm{Zn}$-finger domain of specific transcription factors and thereby impair their activity (Kluska et al., 2020; Tardillo Suárez et al., 2020). The present work exploits the ultimate $50 \times 50 \mathrm{~nm}^{2}$ raster scan resolution of a hard-X-ray synchrotron nanoprobe, and in combination with EM, enables the identification of mitochondria to selectively measure their silver content. When hepatocytes are exposed to silver nanoparticles, we demonstrate a higher accumulation of silver in mitochondria compared to the surrounding cytosol, and a mitophagy process can be observed. This case study illustrates the valuable contribution of correlative imaging to the study of the nano-bio interface, by providing a tool to localize and quantify nanoparticles and trace elements at the organelle level. As such, with an appropriate choice of sample preparation and experimental conditions, this methodology can also be used to address a large panel of questions related to metal homeostasis.

\section{Material and methods}

\section{Cell culture}

HepG2 cells were grown on labtek (Nunc) slides in MEM media supplemented with $10 \%$ Fetal Bovine Serum and $1 \%$ antibiotics-antimycotic at $37^{\circ} \mathrm{C}$ in the presence of $5 \% \mathrm{CO}_{2}$. Cells were exposed to $20 \mathrm{~nm}$ diameter citrate-coated AgNPs, cit-AgNP (Nanocomposix), or to $90 \mathrm{~nm}$ diameter PVP-coated AgNPs, PVP-AgNP (Sigma-Aldrich), at the indicated concentrations of total Ag and for the indicated times. AgNPs were previously characterized (Veronesi et al., 2016). Media and AgNPs were renewed every 24 hours.

\section{Sample preparation for correlative XRF and TEM imaging}

Samples were prepared similarly to our recent work (Tardillo Suárez et al., 2020). At the indicated time points, monolayers of HepG2 cells were fixed overnight at room temperature in a 1:1 ratio mixture of $4 \%$ paraformaldehyde, $0.4 \%$ glutaraldehyde in $0.2 \mathrm{M} \mathrm{PHEM} \mathrm{pH} 7.2$ and culture medium, washed in $0.1 \mathrm{M}$ PHEM pH 7.2, and fixed for 30 minutes in $2 \%$ paraformaldehyde, $0.2 \%$ glutaraldehyde in $0.1 \mathrm{M}$ PHEM pH 7.2, washed in 0.1 M PHEM pH 7.2 and post-fixed in $1 \% \mathrm{OsO}_{4}$ in $0.1 \mathrm{M} \mathrm{PHEM}$ buffer for $1 \mathrm{~h}$ at room temperature. Cells were then dehydrated in graded ethanol series, and flat-embedded using an Epoxy Embedding Medium kit (Sigma-Aldrich). Sections (200 nm) were cut on a Leica UC7 ultra- 
microtome using a DiATOME $35^{\circ}$ diamond knife and collected on 50 nm-thick $\mathrm{Si}_{3} \mathrm{~N}_{4}$ grids (Oxford Instruments).

\section{Transmission electron microscopy}

Digital images were obtained using a Tecnai G2 Spirit BioTwin microscope (FEI) operating at $120 \mathrm{kV}$ with an Orius SC1000 CCD camera (Gatan).

\section{Nano-XRF data acquisition and analysis}

XRF experiments were carried out at the state-of-the-art hard X-ray nanoprobe beamline ID16B at the ESRF (Martínez-Criado et al., 2016). ID16B can be operated in 2 different regimes optimized either for the energy resolution (required for X-ray diffraction and absorption spectroscopy) or for the photon flux (for XRF mapping and holotomography). In the high flux mode used for this experiment, the monochromaticity of the beam $\left(\Delta \mathrm{E} / \mathrm{E}=10^{-2}\right)$ is given by multilayer $\mathrm{KB}$ mirrors and the photon flux is in the $10^{11}$ photons/s range. The incoming photon energy was set at $29.6 \mathrm{keV}$ and the beam was focused down to $55 \times 65 \mathrm{~nm}^{2}$ using Kirkpatrick-Baez mirrors. The fluorescence emission from the sample was recorded using two 3 -element Silicon Drift Detectors (SDD) arrays positioned at $13^{\circ}$ from the sample. High resolution maps were recorded at room temperature, raster scanning the sample in the X-ray focal plane with $50 \times 50 \mathrm{~nm}^{2}$ step size and $500 \mathrm{~ms}$ to $1 \mathrm{~s}$ dwell time/pixel.

Hyperspectral images were analyzed using the PyMCA software package (http://pymca.sourceforge.net/)(Solé et al., 2007). The detector response was calibrated over a thin film multilayer sample from AXO (RF8-200-S2453). XRF data were energy calibrated, normalized by the incoming photon flux, and batch-fitted in order to extract spatially resolved elemental concentrations, assuming a biological matrix of light elements and density of $1 \mathrm{~g} . \mathrm{cm}^{-3}$ according to NIST standards (https://physics.nist.gov/cgi-bin/Star/compos.pl?matno=261). Quantitative results are reported in areal density units $\left(\mathrm{ng} / \mathrm{mm}^{2}\right)$ to facilitate the comparison with previous works. However, considering that in the present case the sample thickness is known, mass fractions $(\mathrm{w} / \mathrm{w})$ can simply be retrieved by dividing areal densities by the assumed sample density $\left(1 \mathrm{~g} . \mathrm{cm}^{-3}\right)$ and by its thickness $(200 \mathrm{~nm})$.

\section{Results and discussion}

\section{Implementation of correlative TEM and XRF microscopy}

Recently, we successfully used XRF on cell sections for the analysis of Ag transformations and distribution within hepatocytes exposed to AgNPs (Tardillo Suárez et al., 2020). In the current study, the 
methodology was extended to be able to pinpoint the trace element content in the organelles of interest. Cells were grown on standard labtek supports that were used for chemical fixation using paraformaldehyde/glutaraldehyde. This method is a good compromise to preserve the intracellular content in comparison to alcohol-based fixation (Jin et al., 2017; Sanchez-Cano et al., 2017). Sample processing was based on classical cell resin-embedding preparation protocols. However, to avoid parasitic signals, all heavy metals classically used to stain and enhance contrast in the sample (for instance $\mathrm{U}$ and $\mathrm{Pb}$ ) were avoided, with the exception of $\mathrm{OsO}_{4}$. The latter is necessary to preserve the cellular ultrastructure by reacting with the lipids in the membranes. Moreover, $\mathrm{OsO}_{4}$ also provides contrast of cellular membranes both in TEM and in XRF, thanks to the electron scattering and an X-ray absorption properties of the heavy Os atoms. The effect of the $\mathrm{OsO}_{4}$ treatment on the elemental content of fixed cells was estimated empirically, by comparing the elemental distributions of metals by XRF imaging in cells exposed to citrate-coated AgNPs for $72 \mathrm{~h}$, then chemically fixed with or without $\mathrm{OsO}_{4}$ post-fixation. The average elemental composition per cell, estimated over three cells per condition, are reported in Table S1. The trace element contents in the cell area were measured, and showed that only in the sample post-fixed with $\mathrm{OsO}_{4}$ it is possible to distinguish basal $\mathrm{Fe}$ from the background, and that the native $\mathrm{Cu}$ content is double that of the Os-free sample. It is not possible however, to determine the amount of native $\mathrm{Zn}$ in the presence of Os, because of the overlap of the X-ray emission lines of the two elements (Fig. S1 and Table S1). Moreover, the cytosolic concentration of molecular Ag(I) determined by XRF mapping is twice as high in the case of hepatocytes prepared with Os in comparison to cells prepared without, $0.22+/-0.04 \mathrm{ng} / \mathrm{mm}^{2}$ versus $0.11+/-0.02 \mathrm{ng} / \mathrm{mm}^{2}$, respectively (Fig. S1). Os fixation is therefore crucial to retain the soluble metal ion complexes inside a cell and its various compartments. A possible explanation resides in the fixation mechanism known for $\mathrm{OsO}_{4}$, which stabilizes the cellular membranes by cross-linking their phospholipidic chains (Wigglesworth, 1957), resulting in the retention of labile ions by cellular compartments. For this reason, and because Os provides contrast of the cellular membranes in XRF, enabling the identification of organelles and a direct comparison with TEM micrographs, we introduced $\mathrm{OsO}_{4}$ fixation in the optimized sample preparation protocol. However, whenever the determination of $\mathrm{Zn}$ content is crucial for the scientific question under investigation, and if the X-ray beam energy is above the Os LII-edge excitation threshold $(10.87 \mathrm{keV})$, another preparation protocol should be applied, by substituting $\mathrm{OsO}_{4}$ with another oxidant acting on lipids for example. Alternatively, the X-ray energy could be set between $9.66 \mathrm{keV}$ (Zn K-absorption edge) and $10.87 \mathrm{keV}$, 
in such a way that only $\mathrm{Zn}$ atoms are excited, whereas Os atoms are not. This last option would not be suitable for the present study, where the energy has to be optimized for the excitation of Ag core-levels (above $25.51 \mathrm{keV}$ ) in order to achieve the highest sensitivity to this metal, but would be pertinent for studies focused on $1^{\text {st }}$ row transition metals. Ideally, the opportunity to map a sample area both at high energy (above the Ag K-edge) and at $\sim 10 \mathrm{keV}$ would allow the most efficient detection of the exogenous metal, of the native trace elements, and a more efficient detection of the lighter physiological elements. This option is often not compatible with the duration of a synchrotron experiment, and a single excitation energy has to be chosen, leading to the choices mentioned above.

Thin sections produced by ultramicrotomy were deposited on $\mathrm{Si}_{3} \mathrm{~N}_{4} \mathrm{EM}$ grids comprising a square window $500 \mu \mathrm{m}$ wide and $50 \mathrm{~nm}$ thick that are suitable for both $\mathrm{EM}$ and XRF imaging. The surface area is limited, which limits the number of cells in the window but facilitates the retrieval of the areas of interest. The $\mathrm{Si}_{3} \mathrm{~N}_{4} \mathrm{EM}$ grid was first scrutinized by TEM to identify cells with organelles and/or markers of interest inside. The position of the chosen cells was roughly found by the optical microscope on the ID16B beamline. Fast XRF mapping at low dose deposition was performed at low spatial resolution with $1 \times 1 \mu \mathrm{m}^{2}$ step size and $100 \mathrm{~ms}$ dwell time to confirm the location of the cells of interest by observing the Os signal. The latter also provided shadows of the organelles and thus gave confidence in the proper retrieval of the area of interest. Fast scans also limited the radiation damage. In a second step, XRF mapping at high-resolution (down to $50 \times 50 \mathrm{~nm}^{2}$ step size and with a dwell time of $500 \mathrm{~ms}$ per point or more) was performed to obtain high quality XRF data in terms of both spatial resolution and sensitivity. These maps were acquired on an area of the order of $10 \times 10 \mu \mathrm{m}^{2}$ in order to limit the acquisition time to a few hours.

A section of an HepG2 cell exposed for 6 hours to $50 \mu \mathrm{M}$ cit-AgNPs was sequentially imaged by TEM (Fig. 1A) and then XRF (Fig. 1B-C). The low-resolution TEM image of the complete cell section revealed the different cellular compartments; among which the nucleus is the largest, and which can also be identified on the XRF map of Os La since the nucleus is not penetrated by this metal species. As a proof of principle, the TEM image enabled to observe electron dense objects (red and orange arrows) that can be attributed to Os deposits (orange arrows) and AgNPs in endosomes or lysosomes (red arrows) thanks to the extraction of the specific elemental maps of Os and Ag, respectively (Fig. 1D-E). Therefore, such a correlative use of TEM and XRF enables the simultaneous determination of the ultrastructure and of the content of the elements of interest in the interior of a cell. Such information can also be 
retrieved using TEM coupled to energy dispersive X-ray spectroscopy. However, the sub-ppm sensitivity of $\mathrm{XRF}$ is necessary to detect trace metal amounts of a few attograms $\left(10^{-18} \mathrm{~g}\right)$ per pixel (Veronesi et al., 2016).
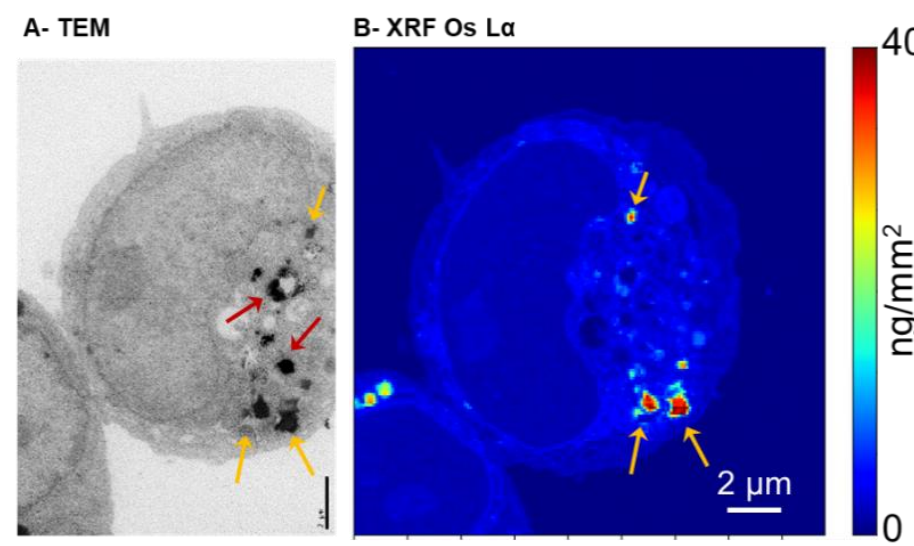

D- EM/XRF Os overlay

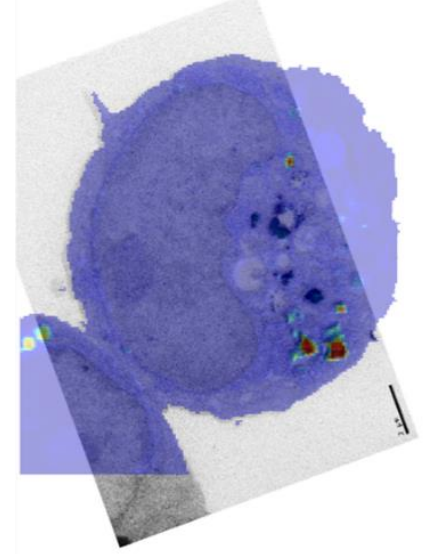

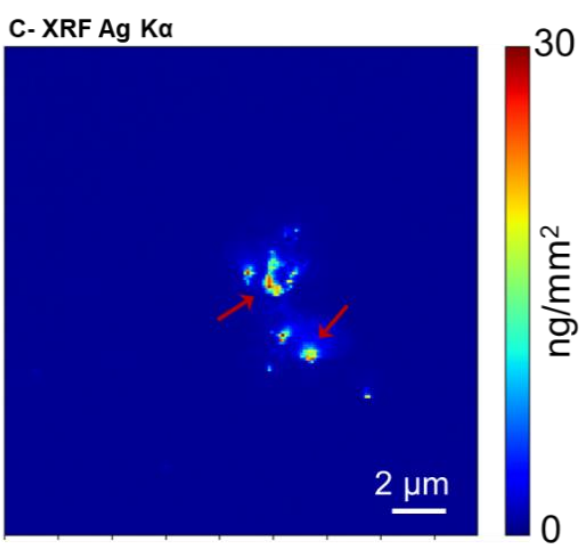

E- EM/XRF Ag overlay

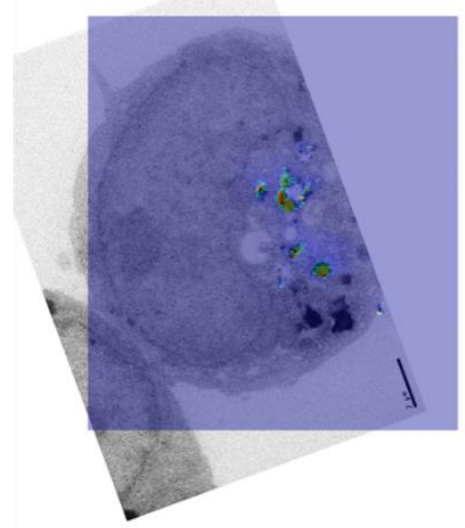

Fig. 1. Correlative TEM and XRF for the identification of Os deposits versus AgNPs. Analysis of a 200 $\mathrm{nm}$ section of a HepG2 cell exposed to $50 \mu \mathrm{M}$ cit-AgNPs for $6 \mathrm{~h}$. (A) TEM image with orange and red arrows pinpointing areas of interest showed in XRF images. (B) Os and (C) $\mathrm{Ag}$ areal density maps in false colors for the same section obtained from XRF data. XRF acquisition was done with a pixel size of $100 \times 100 \mathrm{~nm}^{2}$. TEM micrograph overlayed with Os (D) and Ag (E) XRF maps. All scale bars correspond to $2 \mu \mathrm{m}$.

\section{Sensitivity and spatial resolution of TEM-XRF in comparison to STEM-EDX}

In a recent study, we considered two different chemical forms of silver: i) AgNPs, always found in endosomes and lysosomes, in a partially transformed or in their pristine form, and ii) molecular $\mathrm{Ag}(\mathrm{I})$ thiolate species that were found, at least, in the cytosol or in nuclei (Tardillo Suárez et al., 2020). However, we expect to be able to disclose the distribution of cytosolic Ag between the different organelles such as mitochondria, endoplasmic reticulum or Golgi apparatus. It is frequently assumed that $\mathrm{Ag}(\mathrm{I})$ can accumulate in mitochondria via the $\mathrm{Cu}(\mathrm{I})$ uptake pathway that remains elusive at the molecular level (Cobine et al., 2006). A section of HepG2 cells exposed to $25 \mu \mathrm{M}$ PVP-AgNPs for 24 
hours was inspected by TEM (Fig. 2A). We could easily identify the nucleus surrounded by mitochondria (green rectangle in Fig. 2A and zoom-in in Fig. 2B). The area rich in mitochondria (red rectangle) was selected for further analysis by XRF, with the aim to compare Ag concentration in mitochondria and cytosol. These acquisitions were done at a raster scan resolution of $50 \times 50 \mathrm{~nm}^{2}$ to obtain detailed images of mitochondria. Osmium enabled to confirm the location of mitochondria with respect to the nuclear membrane (Fig. 2C). Very interestingly, the Ag areal density map in false color (Fig. 2D and 2E) revealed a slightly higher concentration of $\mathrm{Ag}$ within mitochondria compared to the surrounding cytosol: $0.08 \mathrm{ng} / \mathrm{mm}^{2}$ in average inside mitochondria versus $0.05 \mathrm{ng} / \mathrm{mm}^{2}$ in the cytosol, after background subtraction and fitting of the XRF signal integrated over the two different cellular compartments. This demonstrates that our analytical protocol is able to map the subcellular distribution of highly diluted dissolved soluble silver species in selected compartments. 


\section{A- TEM large field of view}

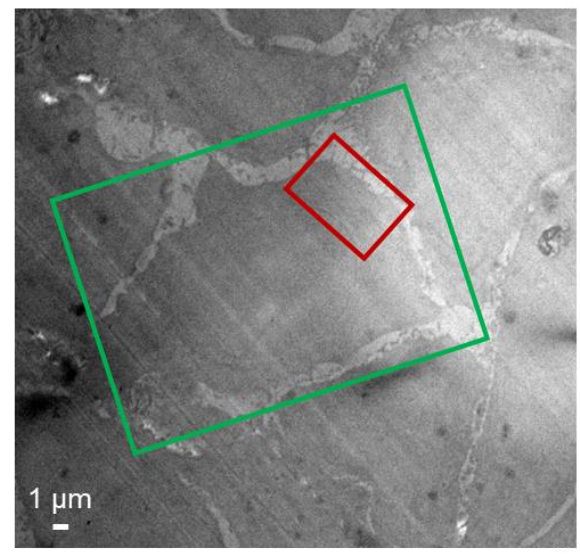

C- XRF Os areal density

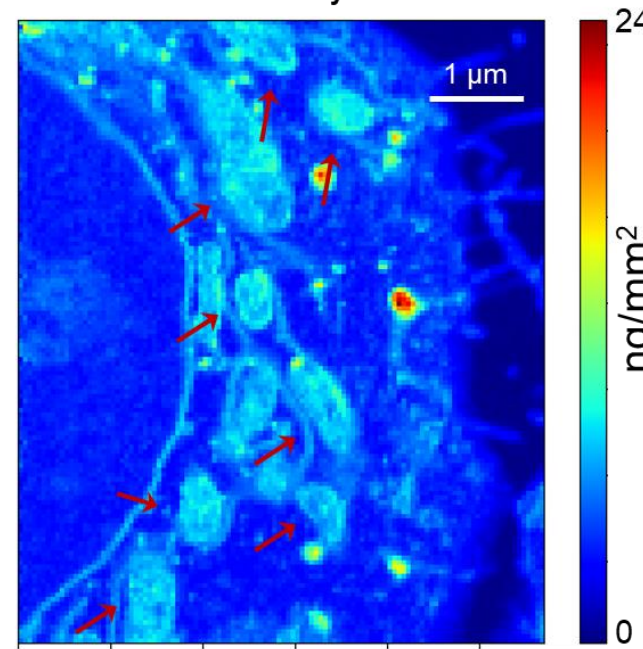

E- EM/XRF Ag overlay

\section{B- Region of interest for XRF}

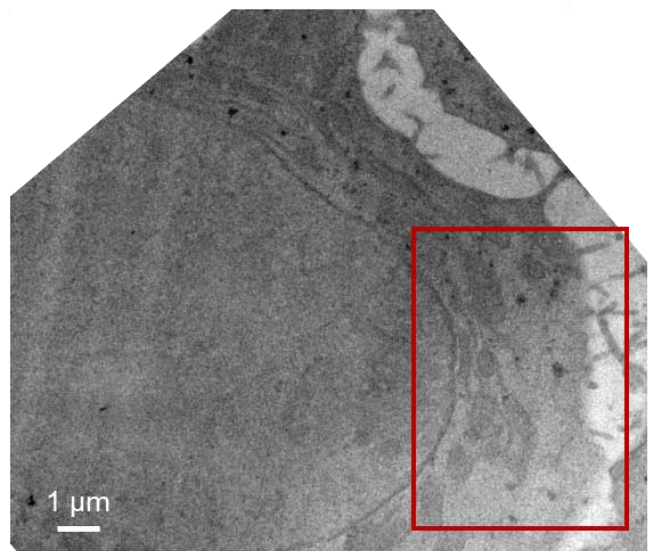

D- XRF Ag areal density

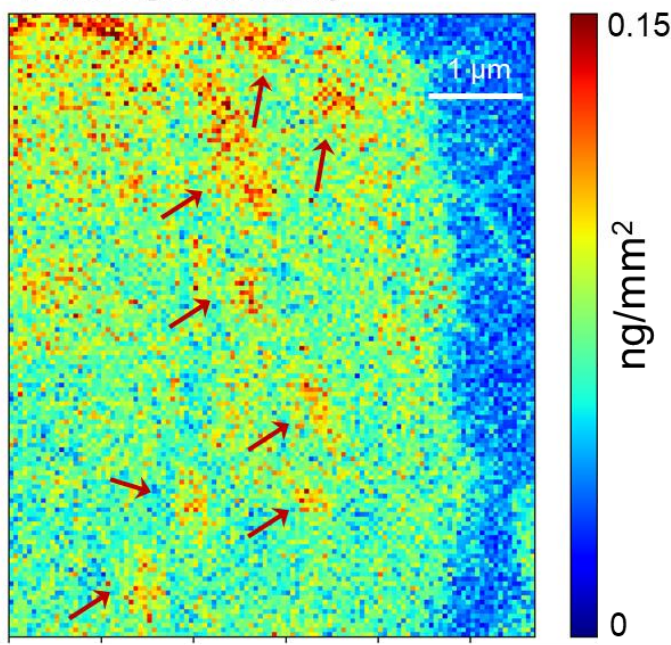

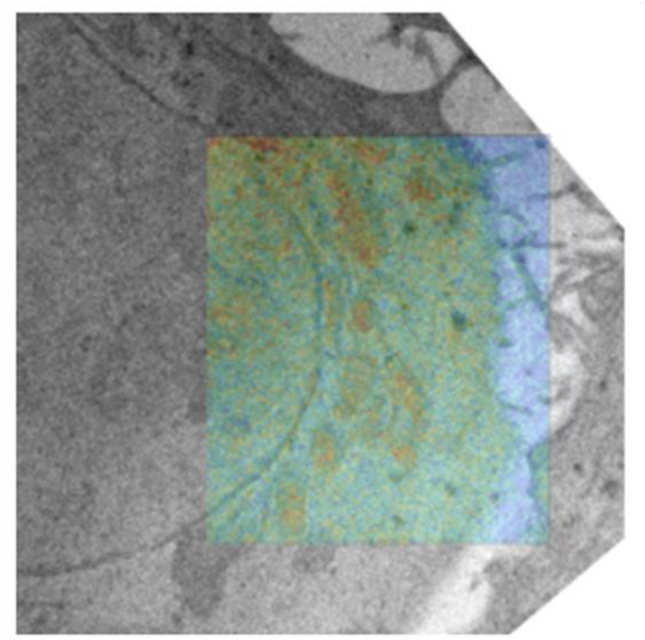

Fig. 2. $\mathrm{Ag}(\mathrm{I})$ accumulation in mitochondria in HepG2 cells exposed to AgNPs. (A) Large TEM field of view with several cells visualized from a section of HepG2 cells exposed to $25 \mu \mathrm{M}$ PVP-AgNPs for 24 hours. (B) Close-up view of the cell of interest, green rectangle in $A$. (C) Os and (D) Ag areal density maps represented in false-colors of the region of interest, red rectangle in $A$ and $B$. Red arrows pinpoint the same mitochondria in $C$ and D. (E) TEM micrograph overlayed with Ag XRF map. XRF acquisitions were done with a pixel size of $50 \times 50 \mathrm{~nm}^{2}$. 
Control cells (i.e. not exposed to AgNPs) were also imaged using this correlative approach (Fig. 3). A field presenting many mitochondria was identified by TEM (Fig. 3A, red rectangle) and then scanned by XRF (Fig. 3B-E). The Os map again revealed mitochondria shadows (Fig. 3B and 3F, red arrows) and the Ag map showed only noise signal (Fig. 3C). The distribution of some native elements in the same field of view is also shown: $\mathrm{Ca}$ (Fig 3D) shows a contrast between the interior and the exterior of the cell, meaning that this native element is detected. However, the high excitation energy chosen to optimize the detection of $\mathrm{Ag}$, and the absorption by the air of the emitted $\mathrm{Ca} \mathrm{K}_{\alpha}$ fluorescence at 3.69 $\mathrm{keV}$, do not allow the quantification of the fine variation of its intracellular concentration. In contrast, the distribution of $\mathrm{Fe}(\mathrm{Fig} \mathbf{3 E})$, as a consequence of the higher energy of the XRF emission of this element (Fe Ka fluorescence at $6.40 \mathrm{keV}$ ), shows a significant signal compared to the background and variations in the local concentration can be correlated with the cell's anatomy. However, none of the native element distributions enabled the localization of mitochondria, for which both Os staining and TEM imaging were necessary.

Altogether, our data demonstrates that the correlative methodology we have developed can efficiently and simultaneously detect AgNPs, soluble silver species, and subcellular compartments. This enabled us to detect minor differences in terms of silver accumulation within a specific organelle, in this case the mitochondria. 


\section{A- TEM large field of view}

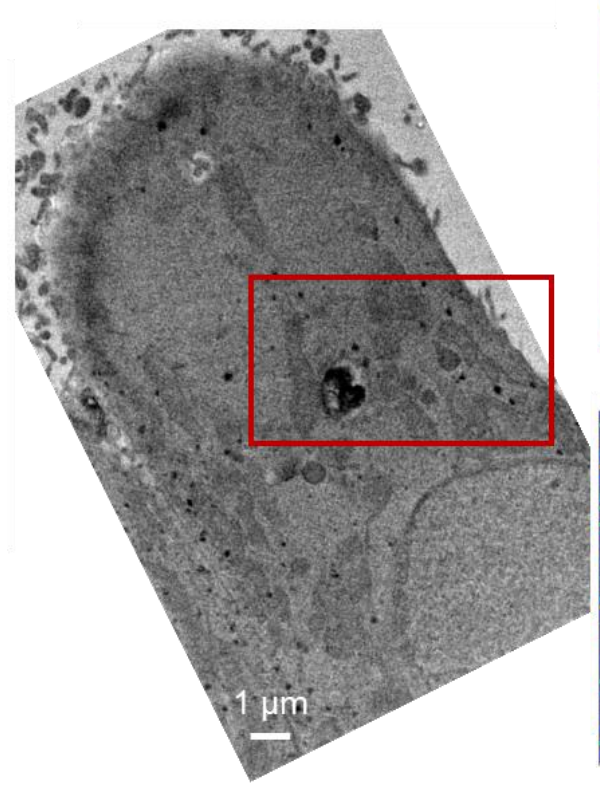

F- EM/XRF Os overlay

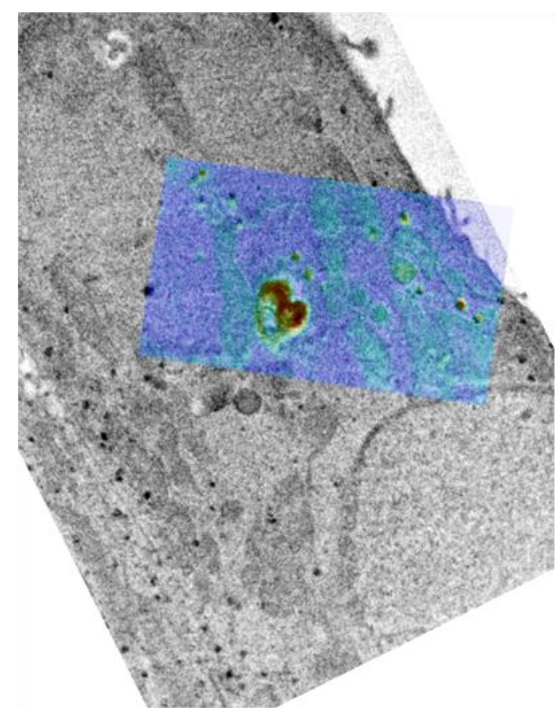

B- XRF Os areal density

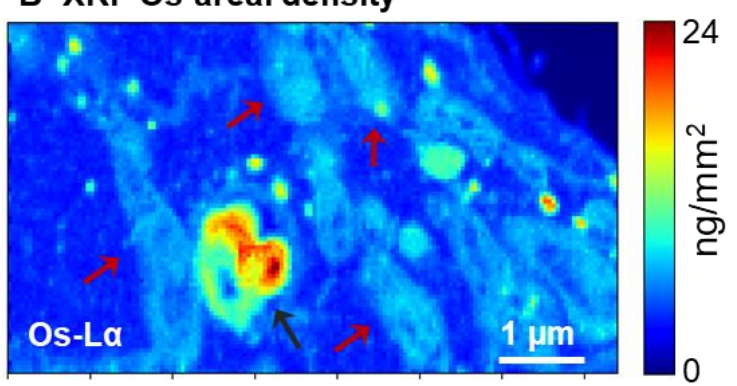

C- XRF Ag areal density

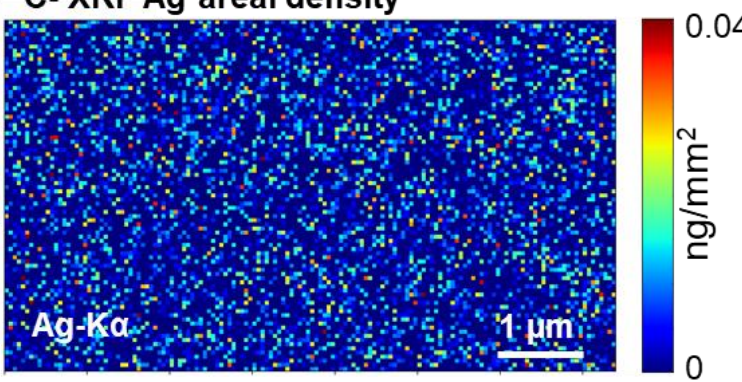

D- XRF Ca areal density

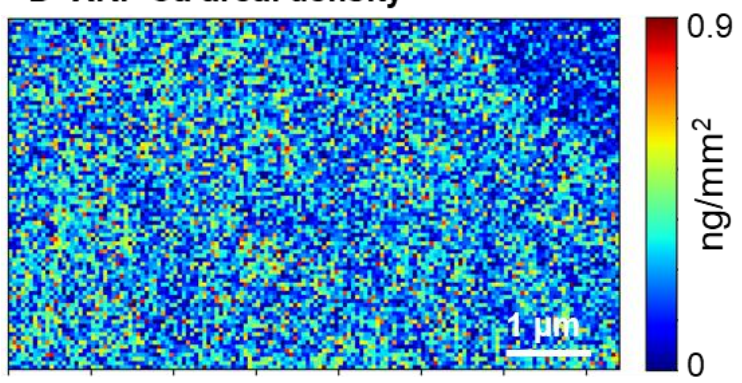

\section{E- XRF Fe areal density}

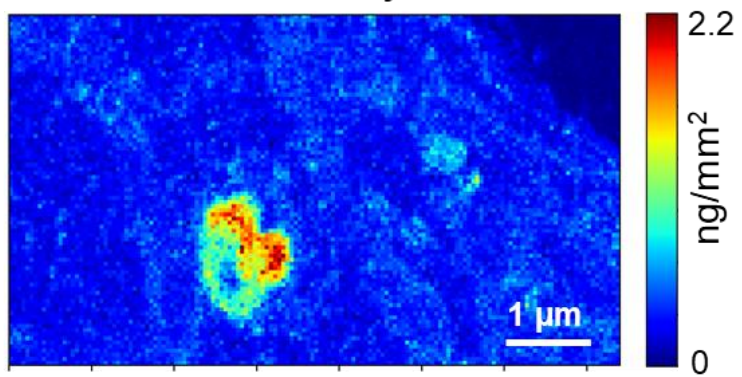

Fig. 3. Control cells analysis. (A) TEM image of the region of interest in a section of HepG2 cells nonexposed to AgNPs. (B) Os, (C) Ag, (D) Ca and (E) Fe areal density maps in false colors for the same region of interest, red rectangle in A. Mitochondrial shadows are pinpointed with red arrows in B. Black arrow pinpoint a vesicle containing Os deposit as seen by TEM (A). (F) TEM micrograph overlayed with Os XRF map. XRF acquisition was done with a pixel size of $50 \times 50 \mathrm{~nm}^{2}$.

To compare this multimodal approach with conventional STEM-EDX, a field comprising part of a nuclei, cytosol, a mitochondria (red arrow), and a lysosome containing AgNPs and transformed AgNPs (orange arrow) was imaged by STEM (Fig. S2A-B). EDX analysis showed that only electron dense spots within the lysosome present a significant Ag signal (Fig. S2C-D), confirming that these were AgNPs or dissolving AgNPs. The mitochondria, the cytosol and the nucleus did not display a detectable Ag signal 
by EDX, while synchrotron nanoprobe XRF enabled us not only to detect molecular $\mathrm{Ag}(\mathrm{I})$ species found in these three compartments but also to precisely quantify local $\mathrm{Ag}(\mathrm{I})$ concentrations and thus rank them according to their $\mathrm{Ag}(\mathrm{I})$ accumulation: the highest is found in mitochondria, followed by the cytosol and finally the nucleus. This is an empirical demonstration of the higher sensitivity of SR-XRF with respect to STEM-EDX, as already reported in the literature (Decelle et al., 2020). In order to explain the fundamental reasons for this difference, we have to consider that both STEM-EDX and SR-XRF rely on the physical principle of the photoionization of core electrons of the atoms in the specimen, followed by the emission of characteristic $\mathrm{X}$-rays due to their radiative de-excitation. The two techniques differ in the choice of the probe: synchrotron X-rays in SR-XRF, electrons in STEM-EDX. The observed higher sensitivity of the former most probably depends on the higher efficiency of the excitation operated by Xrays with respect to electrons. The theoretical description of these phenomena could help elucidate the difference between the sensitivity of the two techniques, as in the literature such a comparison relies only on empirical basis. These calculations are beyond the scope of this work, and a discussion meant to set the basis for it, is reported in the SI.

In conclusion, the combined ultrastructural imaging and trace element quantification obtained by the correlative TEM and XRF imaging approach provides additional information with respect to the STEMEDX method.

\section{XRF offers a high dynamic range}

Together with sensitivity to low concentrations, the dynamic range is an important parameter in elemental imaging, in particular for elements that are present in both their crystalline form (e.g. nanoparticles) and as soluble molecular complexes. An example in biology is Fe, which is stored in a mineral form in ferritin and exists also as an enzyme co-factor. The dynamic range is also crucial in the determination of the intracellular fate of AgNP, where both crystalline and soluble silver need to be detected. Indeed, AgNPs are endocytosed and dissolved within endo-lysosomes into $\mathrm{Ag}(\mathrm{I})$ species that are then distributed throughout the cell (Veronesi et al., 2016). XRF is quantitative over at least 5 orders of magnitude, as shown in Fig. 4. XRF rendering using a linear scale ranging from 0 to $80 \mathrm{ng} / \mathrm{mm}^{2}$ only highlights the presence of Ag-rich spots in vesicles, attributable to internalized AgNPs, together with a much weaker signal throughout the vesicles, most probably due to $\mathrm{Ag}(\mathrm{I})$ released from the NPs in the acidic lysosomal environment (Fig. 4B, red arrows). However, the visualization of Ag in the same area 
with a scale ranging between 0 and $0.5 \mathrm{ng} / \mathrm{mm}^{2}$ highlights the presence of a much weaker signal $(<0.1$ $\mathrm{ng} / \mathrm{mm}^{2}$ ) in the cell cytosol, not visible in the previous representations (Fig. 4C). Finally, the use of a logarithmic-scale representation of Ag distribution highlights at a glance the presence of all Ag species, regardless of their concentration (Fig. 4D). The detection of these species is made possible by the choice of the excitation energy, optimized for the photoexcitation of silver core electrons (Ag K-edge), and by the high photon flux of $10^{11}$ photons/s provided by the nanoprobe in the chosen experimental conditions.
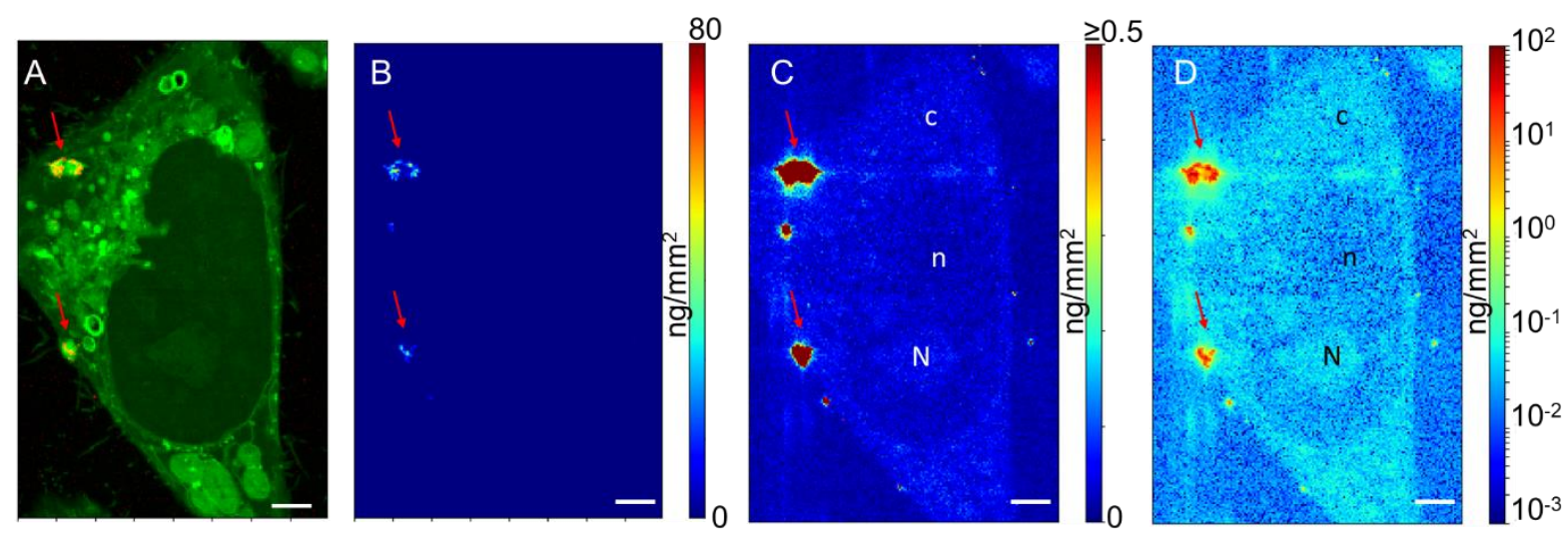

Fig. 4. False-color representation of $\mathrm{Ag}$ distribution in hepatic cells exposed to $50 \mu \mathrm{M}$ citrate-coated AgNPs for $72 \mathrm{~h}$. (A) Os (green) and Ag (red) two-color map highlights the subcellular compartments and the presence of Ag-rich vesicles (red arrows). (B) Ag distribution visualized using a linear color scale ranging between 0 and $80 \mathrm{ng} / \mathrm{mm}^{2}$. (C) Ag distribution visualized using a linear color scale ranging between 0 and $0.5 \mathrm{ng} / \mathrm{mm}^{2}$. (D) Logarithmic-scale representation of $\mathrm{Ag}$ distribution in the same area (0$100 \mathrm{ng} / \mathrm{mm}^{2}$ ). Red arrows pinpoint endo-lysosomes containing partially transformed AgNP. $\mathrm{n}, \mathrm{N}$ and c stand for nucleus, nucleolus and cytosol, respectively. Scale bars correspond to $2 \mu \mathrm{m}$.

This example demonstrates that XRF nano-imaging has the unique advantage to be able to detect simultaneously metallic nanoparticles and soluble metal complexes, providing high detection performances over a very large concentration range. It also highlights the importance of the choice of the visualization parameters to reveal the presence of all signals detected by the hyperspectral imaging technique. Finally, the high sensitivity and dynamic range of SR-XRF are interesting to correlate with EM ultrastructure imaging only because progress has recently been made in its spatial resolution which is now getting closer to that of EM.

\section{Catching events, the example of mitochondrial Ag overload-induced mitophagy}

One main advantage of a single-cell, imaging-based analysis method compared to a bulk quantification approach is the possibility to identify specific events that occur rarely, but that are crucial to completely 
understand a biological process. Moreover, an imaging method can also dissect a whole process, by visualizing the whole cascade of events. In the data acquired on HepG2 cells exposed to $25 \mu \mathrm{M}$ PVPAgNPs for 72 hours, a mitochondrion located inside a double membrane vesicle was visualized by TEM (Fig. 5A, red arrow). This is clearly a mitochondrion that has been engulfed in an autophagosome, illustrating the triggering of a mitophagy process (Ding and Yin, 2012). The Ag areal density map of this region revealed a high Ag content in the mitochondrion located in the autophagosome (Fig. 5B-C, red arrow). Indeed, the value was in the order of $1 \mathrm{ng} / \mathrm{mm}^{2}$, to be compared to the typical 0.04 to 0.14 $\mathrm{ng} / \mathrm{mm}^{2}$ range observed in normal mitochondria in the various tested conditions. Therefore, this particular mitochondrion accumulated 10-times more $\mathrm{Ag}$ than healthy mitochondria. This example highlights the importance of associating quantitative elemental distributions to high-resolution structural observations, since it revealed that the observed mitophagy is likely due to Ag overload. Current cell analyses assume comparable $\mathrm{Ag}(\mathrm{I})$ molecular species uptake for a type of organelle and previous studies showed directly or indirectly a significant $A g(I)$ entry into mitochondria (Vest et al., 2013). However, our data showed a broad variability in silver amounts accumulated in each of the mitochondria. Interestingly, close to this mitochondrion undergoing mitophagy, Ag hot spots could be observed in Fig. 5B-C (red pixels), corresponding to AgNPs and/or transformed AgNPs, as confirmed by the TEM micrograph (Fig. 5A and $\mathbf{5 C}$ ). It is thus possible to imagine that mitochondria close to endosomes and/or lysosomes containing dissolving AgNPs undergo a massive accumulation of Ag that leads them to collapse and be degraded by mitophagy.
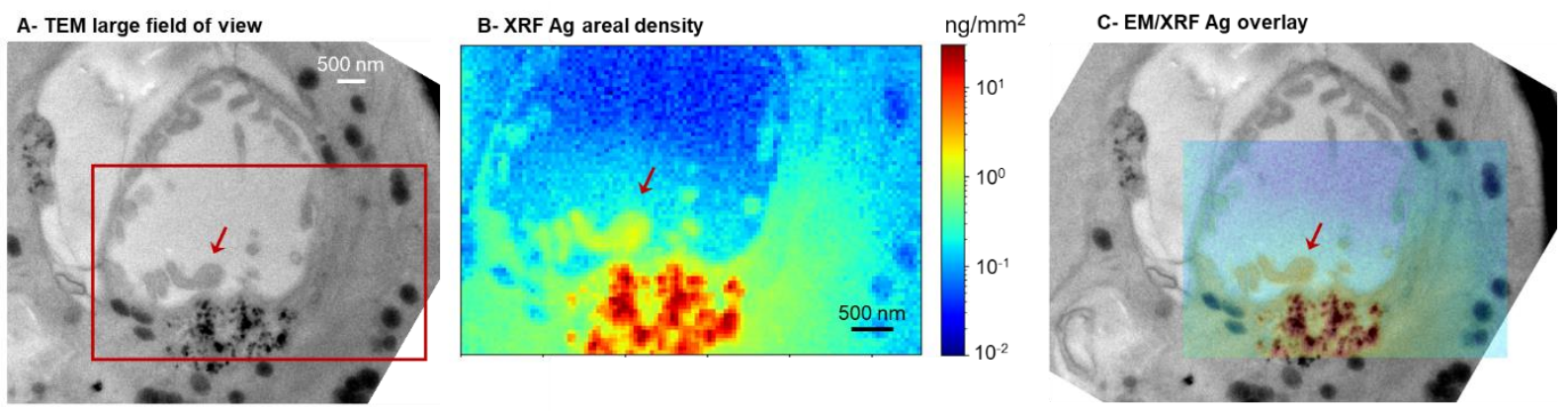

Fig. 5. Ag-overload induce mitophagy. (A) TEM image from a section of HepG2 cells exposed to $25 \mu \mathrm{M}$ PVP-AgNPs for 72 hours. (B) Ag areal density map in false colors of the region of interest, red rectangle in A. (C) TEM micrograph overlayed with Ag XRF map. The red arrow pinpoints the same mitochondria in $A, B$ and $C$. XRF acquisition was done with a pixel size of $50 \times 50 \mathrm{~nm}^{2}$.* 


\section{Conclusions}

In the current study, we have developed correlative TEM and hard X-ray nanoprobe XRF imaging on the same cell section, by using a preparation protocol for classical cellular $\mathrm{EM}$, modified to use $\mathrm{OsO}_{4}$ as the only heavy metal staining. Indeed, $\mathrm{OsO}_{4}$ is required both to protect trace elements from partial leakage out of the cell and to visualize cellular membranes where Os accumulates. This methodology has been optimized on sections of $200 \mathrm{~nm}$. This thickness was chosen to provide, on the one hand, TEM images with enough detail to identify the different organelles, and on the other hand, high enough XRF signal for trace element mapping. The synergistic use of the two techniques on the same sample enables to map selected elemental distributions at the organelle level. The quality of the data that can be obtained, as well as the sensitivity and the accuracy of the quantification, were evidenced in this study by the analysis of $\mathrm{Ag}(\mathrm{I})$ molecular species partitioning between mitochondria and the surrounding cytosol in hepatocytes that have been exposed to AgNPs. These experiments proved that, following AgNP dissolution into $\mathrm{Ag}(\mathrm{I})$, molecular species containing $\mathrm{Ag}(\mathrm{I})$ can enter mitochondria. The entry could be due to the recruitment of $\mathrm{Ag}(\mathrm{I})$ by the $\mathrm{Cu}(\mathrm{l})$ mitochondrial uptake pathway. However, this process seems rather not controlled by the cell since mitochondrial uptake of $\mathrm{Ag}(\mathrm{I})$ can lead to a mitochondrion with ten times higher level than all others, ending up into an autophagosome. This event was observed close to a lysosome containing dissolving AgNPs. One can hypothesize that $\mathrm{Ag}(\mathrm{I})$ uptake is mainly driven by localization effect and no other control mechanism exists. All these data bring insight in the context of AgNP fate and toxicity in the liver, and demonstrate the usefulness of the method.

From a more general standpoint, with the opportune choice of the X-ray excitation energy and sample preparation protocol, this method can be extended to the study at the subcellular level of mechanisms involving other trace elements, such as iron or copper homeostasis. In this study, we chose a beam energy of $29.6 \mathrm{keV}$, which is optimal for Ag mapping but not for the detection of the lighter, native metals such as $\mathrm{Cu}$ or $\mathrm{Zn}$, for which a lower excitation energy is required to obtain the highest sensitivity. Moreover, the Os L edge can overlap with some elements of interest, for instance $\mathrm{Zn}$. To overcome this limitation, the sample preparation could be modified by replacing $\mathrm{OsO}_{4}$ with $\mathrm{RuO}_{4}$-(Swartzendruber et al., 1995). Finally, it is worth mentioning that the ESRF, as well as other synchrotrons world-wide, recently upgraded their storage ring to obtain enhanced performances. On the beamline ID16B, this originates a 10-fold increase in the photon flux, which will enable faster acquisition time in the ms/pixel range, provided that $\mathrm{XRF}$ detectors can run in this regime. Reducing the acquisition time will also be 
necessary to preserve the samples from beam-induced damages. Combined to the pre-screening of the samples through TEM and pre-identification of the regions of interest, SR-XRF imaging can provide elemental compositions with unrivalled sensitivity, resolution, and acquisition time.

In conclusion, the correlative TEM and XRF imaging approach presented here consists in optimizing the implemented use of the two techniques, commonly used separately, to obtain elemental distribution maps enriched with their ultrastructural context, which is crucial for the study of metal homeostasis mechanisms or to understand the fate of non-physiological metals.

\section{Conflicts of interest.}

The authors have no conflicts of interest to disclose.

\section{Acknowledgements}

The authors acknowledge the ESRF for providing beam time on the beamline ID16B-NA. This work used the platforms of the Grenoble Instruct-ERIC Center (ISBG: UMS 3518 CNRS-CEA-UGA-EMBL) with support from FRISBI (ANR-10-INSB-05-02) and GRAL (ANR-10-LABX-49-01) within the Grenoble Partnership for Structural Biology (PSB). The EM facility is headed by Guy Schoehn and supported by the Rhône-Alpes Region, the Fondation Recherche Medicale (FRM), the fonds FEDER and the GISInfrastrutures en Biologie Sante et Agronomie (IBISA). This project received funding from GRAL and Arcane Labex, a programme from the Chemistry Biology Health $(\mathrm{CBH})$ Graduate School of University Grenoble Alpes (ANR-17-EURE-0003). This research is also part of the LabEx SERENADE (grant ANR11-LABX-0064).

\section{References}

Beilschmidt, L.K., Ollagnier de Choudens, S., Fournier, M., Sanakis, I., Hograindleur, M.-A., Clémancey, M., Blondin, G., Schmucker, S., Eisenmann, A., Weiss, A., Koebel, P., Messaddeq, N., Puccio, H., Martelli, A., 2017. ISCA1 is essential for mitochondrial Fe4S4 biogenesis in vivo. Nat. Commun. 8, 15124. https://doi.org/10.1038/ncomms 15124

Busser, B., Moncayo, S., Coll, J.-L., Sancey, L., Motto-Ros, V., 2018. Elemental imaging using laserinduced breakdown spectroscopy: A new and promising approach for biological and medical applications. Coord. Chem. Rev. 358, 70-79. https://doi.org/10.1016/j.ccr.2017.12.006

Carmona, A., Zogzas, C.E., Roudeau, S., Porcaro, F., Garrevoet, J., Spiers, K.M., Salomé, M., Cloetens, P., Mukhopadhyay, S., Ortega, R., 2019. SLC30A10 Mutation Involved in Parkinsonism Results in Manganese Accumulation within Nanovesicles of the Golgi Apparatus. ACS Chem. Neurosci. 10, 599-609. https://doi.org/10.1021/acschemneuro.8b00451 
Chen, K.G., Valencia, J.C., Lai, B., Zhang, G., Paterson, J.K., Rouzaud, F., Berens, W., Wincovitch, S.M., Garfield, S.H., Leapman, R.D., Hearing, V.J., Gottesman, M.M., 2006. Melanosomal sequestration of cytotoxic drugs contributes to the intractability of malignant melanomas. Proc. Natl. Acad. Sci. 103, 9903-9907. https://doi.org/10.1073/pnas.0600213103

Ciriolo, M.R., Civitareale, P., Carrì, M.T., De Martino, A., Galiazzo, F., Rotilio, G., 1994. Purification and characterization of $\mathrm{Ag}, \mathrm{Zn}$-superoxide dismutase from Saccharomyces cerevisiae exposed to silver. J. Biol. Chem. 269, 25783-25787.

Cobine, P.A., Pierrel, F., Bestwick, M.L., Winge, D.R., 2006. Mitochondrial matrix copper complex used in metallation of cytochrome oxidase and superoxide dismutase. J. Biol. Chem. 281, 3655236559. https://doi.org/10.1074/jbc.M606839200

de Jonge, M.D., Holzner, C., Baines, S.B., Twining, B.S., Ignatyev, K., Diaz, J., Howard, D.L., Legnini, D., Miceli, A., McNulty, I., Jacobsen, C.J., Vogt, S., 2010. Quantitative 3D elemental microtomography of Cyclotella meneghiniana at $400-\mathrm{nm}$ resolution. Proc. Natl. Acad. Sci. 107, 15676-15680. https://doi.org/10.1073/pnas.1001469107

Decelle, J., Veronesi, G., Gallet, B., Stryhanyuk, H., Benettoni, P., Schmidt, M., Tucoulou, R., Passarelli, M., Bohic, S., Clode, P., Musat, N., 2020. Subcellular Chemical Imaging: New Avenues in Cell Biology. Trends Cell Biol. 30, 173-188. https://doi.org/10.1016/j.tcb.2019.12.007

Deng, J., Vine, D.J., Chen, S., Jin, Q., Nashed, Y.S.G., Peterka, T., Vogt, S., Jacobsen, C., 2017. X-ray ptychographic and fluorescence microscopy of frozen-hydrated cells using continuous scanning. Sci. Rep. 7. https://doi.org/10.1038/s41598-017-00569-y

Ding, W.-X., Yin, X.-M., 2012. Mitophagy: mechanisms, pathophysiological roles, and analysis. Biol. Chem. 393, 547-564. https://doi.org/10.1515/hsz-2012-0119

Dressler, V.L., Müller, E.I., Pozebon, D., 2018. Bioimaging Metallomics, in: Arruda, M.A.Z. (Ed.), Metallomics. Springer International Publishing, Cham, pp. 139-181. https://doi.org/10.1007/978-3-319-90143-5_7

Fus, F., Yang, Y., Lee, H.Z.S., Top, S., Carriere, M., Bouron, A., Pacureanu, A., da Silva, J.C., Salmain, M., Vessières, A., Cloetens, P., Jaouen, G., Bohic, S., 2019. Intracellular Localization of an Osmocenyl-Tamoxifen Derivative in Breast Cancer Cells Revealed by Synchrotron Radiation X-ray Fluorescence Nanoimaging. Angew. Chem. Int. Ed. 58, 3461-3465. https://doi.org/10.1002/anie.201812336

Greenhalgh, C.J., Karekla, E., Miles, G.J., Powley, I.R., Costa, C., de Jesus, J., Bailey, M.J., Pritchard, C., MacFarlane, M., Pringle, J.H., Managh, A.J., 2020. Exploration of Matrix Effects in Laser Ablation Inductively Coupled Plasma Mass Spectrometry Imaging of Cisplatin-Treated Tumors. Anal. Chem. 92, 9847-9855. https://doi.org/10.1021/acs.analchem.0c01347

Hasna, J., Bohic, S., Lemoine, S., Blugeon, C., Bouron, A., 2019. Zinc Uptake and Storage During the Formation of the Cerebral Cortex in Mice. Mol. Neurobiol. 56, 6928-6940. https://doi.org/10.1007/s12035-019-1581-7

Jin, Q., Paunesku, T., Lai, B., Gleber, S.-C., Chen, S., Finney, L., Vine, D., Vogt, S., Woloschak, G., Jacobsen, C., 2017. Preserving elemental content in adherent mammalian cells for analysis by synchrotron-based x-ray fluorescence microscopy: PRESERVING ELEMENTAL CONTENT. J. Microsc. 265, 81-93. https://doi.org/10.1111/jmi.12466

Kashiv, Y., Austin, J.R., Lai, B., Rose, V., Vogt, S., El-Muayed, M., 2016. Imaging trace element distributions in single organelles and subcellular features. Sci. Rep. 6. https://doi.org/10.1038/srep21437

Kluska, K., Peris-Díaz, M.D., Płonka, D., Moysa, A., Dadlez, M., Deniaud, A., Bal, W., Krężel, A., 2020. Formation of highly stable multinuclear $\mathrm{Ag} \mathrm{n}_{\mathrm{n}}$ clusters in zinc fingers disrupts their structure and function. Chem. Commun. 56, 1329-1332. https://doi.org/10.1039/C9CC09418K 
Martínez-Criado, G., Villanova, J., Tucoulou, R., Salomon, D., Suuronen, J.-P., Labouré, S., Guilloud, C., Valls, V., Barrett, R., Gagliardini, E., Dabin, Y., Baker, R., Bohic, S., Cohen, C., Morse, J., 2016. ID16B: a hard X-ray nanoprobe beamline at the ESRF for nano-analysis. J. Synchrotron Radiat. 23, 344-352. https://doi.org/10.1107/S1600577515019839

Sanchez-Cano, C., Romero-Canelón, I., Yang, Y., Hands-Portman, I.J., Bohic, S., Cloetens, P., Sadler, P.J., 2017. Synchrotron X-Ray Fluorescence Nanoprobe Reveals Target Sites for OrganoOsmium Complex in Human Ovarian Cancer Cells. Chem. - Eur. J. 23, 2512-2516. https://doi.org/10.1002/chem.201605911

Solé, V.A., Papillon, E., Cotte, M., Walter, P., Susini, J., 2007. A multiplatform code for the analysis of energy-dispersive X-ray fluorescence spectra. Spectrochim. Acta Part B At. Spectrosc. 62, 6368. https://doi.org/10.1016/j.sab.2006.12.002

Swartzendruber, D.C., Burnett, I.H., Wertz, P.W., Madison, K.C., Squier, C.A., 1995. Osmium Tetroxide and Ruthenium Tetroxide Are Complementary Reagents for the Preparation of Epidermal Samples for Transmission Electron Microscopy. J. Invest. Dermatol. 104, 417-420. https://doi.org/10.1111/1523-1747.ep12665909

Tardillo Suárez, V., Karepina, E., Chevallet, M., Gallet, B., Cottet-Rousselle, C., Charbonnier, P., Moriscot, C., Michaud-Soret, I., Bal, W., Fuchs, A., Tucoulou, R., Jouneau, P.-H., Veronesi, G., Deniaud, A., 2020. Nuclear translocation of silver ions and hepatocyte nuclear receptor impairment upon exposure to silver nanoparticles. Environ. Sci. Nano 7, 1373-1387. https://doi.org/10.1039/C9EN01348B

Veronesi, G., Deniaud, A., Gallon, T., Jouneau, P.-H., Villanova, J., Delangle, P., Carrière, M., Kieffer, I., Charbonnier, P., Mintz, E., Michaud-Soret, I., 2016. Visualization, quantification and coordination of $\mathrm{Ag}+$ ions released from silver nanoparticles in hepatocytes. Nanoscale 8, 17012-17021. https://doi.org/10.1039/c6nr04381j

Vest, K.E., Leary, S.C., Winge, D.R., Cobine, P.A., 2013. Copper import into the mitochondrial matrix in Saccharomyces cerevisiae is mediated by Pic2, a mitochondrial carrier family protein. J. Biol. Chem. 288, 23884-23892. https://doi.org/10.1074/jbc.M113.470674

Wiemann, M., Vennemann, A., Blaske, F., Sperling, M., Karst, U., 2017. Silver Nanoparticles in the Lung: Toxic Effects and Focal Accumulation of Silver in Remote Organs. Nanomaterials 7, 441. https://doi.org/10.3390/nano7120441

Wigglesworth, V.B., 1957. The use of osmium in the fixation and staining of tissues. Proc. R. Soc. Lond. Ser. B - Biol. Sci. 147, 185-199. https://doi.org/10.1098/rspb.1957.0043

Yuan, Y., Chen, S., Paunesku, T., Gleber, S.C., Liu, W.C., Doty, C.B., Mak, R., Deng, J., Jin, Q., Lai, B., Brister, K., Flachenecker, C., Jacobsen, C., Vogt, S., Woloschak, G.E., 2013. Epidermal Growth Factor Receptor Targeted Nuclear Delivery and High-Resolution Whole Cell X-ray Imaging of $\mathrm{Fe}_{3} \mathrm{O}_{4} @ \mathrm{TTiO}_{2}$ Nanoparticles in Cancer Cells. ACS Nano 7, 10502-10517. https://doi.org/10.1021/nn4033294 